\title{
IL POETA TRA LA FOLLA DELLA ROMA DI AUGUSTO
}

\author{
Luigi Galasso* \\ Recebido em: 30/10/2018 \\ Aprovado em: 09/11/2018
}

* Università Cattolica del Sacro Cuore, Milano. luigi.galasso@unicatt.it

RIASSUNTO: I poeti augustei hanno un'attitudine complessa nei confronti delle cerimonie pubbliche. In alcune delle sue $O d i$, soprattutto del IV libro, Orazio vuole essere visto come uno tra molti nella sua celebrazione del princeps. Si tratta di componimenti con molti livelli di significato, che mostrano un' interessante dialettica tra l'aspetto pubblico e quello privato ed un particolare rapporto con i modelli poetici greci, arcaici ed ellenistici. Ovidio sviluppa questi spunti nelle sue elegie dell'esilio per dare vita ad una nuova poesia panegiristica.

PAROLE CHIAVE: Orazio; poesia pubblica; Pindaro; Ovidio; esilio.

THE POET AMONG THE CROWD IN AUGUSTAN ROME

\begin{abstract}
Augustan poets have a complex attitude towards public ceremonies. In some of his Odes, especially in the fourth book, Horace wants to be seen as one among many in his celebration of the princeps. These are multi-layered poems, which show a subtle dialectic public/ private and an interesting relationship with Lyric and Hellenistic Greek models. Ovid develops these suggestions in his exile elegies to give life to a new panegyrical poetry.
\end{abstract}

KEYWORDS: Horace; public poetry; Pindar; Ovid; exile.

\footnotetext{
11 rma deus Caesar dives meditatur ad Indos, "Il divo Cesare si prepara a combattere i ricchi Indi", apre l'elegia 4 del III libro di Properzio: egli prefigura la grande spedizione di Augusto in Oriente, che sarà senz'altro coronata dal successo, e si augura di poter assistere al trionfo (3.4.15-6):
} 
inque sinu carae nixus spectare puellae incipiam et titulis oppida capta legam. ${ }^{1}$

poggiato sul seno della ragazza diletta comincerò a guardare e leggerò sulle tavolette i nomi delle città.

Il poeta elegiaco naturalmente non partecipa alle imprese militari. Ma cosa significa ammirare il trionfo appoggiato sul seno della donna amata? Possiamo pensare che ci sia la volontà di collocarsi in una posizione distaccata. Non si esprime aggressività, ma distacco, coerentemente con il carattere delle prime elegie del III libro - sono i componimenti che sono stati definiti il corrispettivo delle Odi romane. ${ }^{2}$ Properzio, come un vates, ha profetizzato il trionfo di Augusto e anche, come augur, ha tratto auspici favorevoli all'impresa: omina fausta cano (v. 10). ${ }^{3}$

La scena dei due amanti porta con naturalezza a Venere, a cui è rivolta la preghiera per il princeps, che da lei discende, e per il popolo di Roma. Nell'ultimo distico (v. 21-2) chi si avvia alla spedizione ed è destinato a meritare il bottino ${ }^{4}$ viene contrapposto al poeta, per il quale sarà sufficiente applaudire lungo la via Sacra.

La presenza di Cinzia nel momento della celebrazione del princeps fa sì che Properzio possa rimanere pur sempre all'interno del suo mondo. In questo senso è significativo il confronto con la situazione messa in scena da Cornelio Gallo (3 Blänsdorf, 2 Courtney, 145 Hollis):
Fata mihi, Caesar, tum erunt mea dulcia, quom tu maxima Romanae pars eri $<$ s $>$ historiae
postque tuum reditum multorum templa deorum fixa legam spolieis deivitiora tueis.
Il mio destino allora sarà felice, quando tu, o Cesare, sarai la parte maggiore della storia romana,
e dopo il tuo ritorno passerò in rassegna i templi di molti dèi resi più ricchi dalle spoglie che vi sono appese.

Al testo di Gallo con ogni probabilità Properzio allude, ${ }^{5}$ ma c'è una differenza sostanziale: per Properzio il trionfo è semplicemente uno spettacolo da ammirare dall'esterno,

\footnotetext{
${ }^{1}$ La ricollocazione dei versi nell'edizione di Stephen Heyworth (2007), argomentata in Heyworth, 2007, p. 295-7, rende il distico ancora più denso di significato.

${ }^{2}$ Fedeli, 1985, p. 33.

${ }^{3}$ Fedeli, 1985, p. 165.

${ }^{4}$ L'insistenza sulla preda è significativa, messa com'è in rilievo all'inizio e alla fine, e da ciò il poeta elegiaco prende le distanze: cf. Putnam, 1986, p. 58-9. A questo si aggiunge, naturalmente, il motivo legittimo della guerra che vuole vendicare il disonore della sconfitta di Crasso.

${ }^{5}$ Putnam, 1980, p. 49-56; cf. anche Giangrande, 1980, p. 141-53: il rifiuto della paternità di Gallo per i versi del papiro (tesi riproposta in "On the Pseudo-Gallus", in Giangrande, 1982, p. 83-93)
} 
rimanendo chiusi nel proprio mondo, mentre per Gallo la vittoria di Cesare (in questo caso si tratta di Giulio Cesare) produce un cambiamento nella propria vita. Il destino di dolore del poeta d'amore diverrà paradossalmente lieto in occasione della vittoria del suo condottiero, che sarà parte grande della storia di Roma: fata mibi tum erunt mea dulcia. ${ }^{6}$

In simili circostanze è particolarmente rivelatore l'atteggiamento del singolo, e specialmente del singolo autore, che si deve confrontare con la poesia celebrativa - anche se solo implicitamente, come accade con i poeti del circolo di Mecenate. Non si può rimanere indifferenti, del resto, in occasione delle grandi cerimonie in cui il sovrano mette in scena se stesso. Questo valeva già per i Tolemei ad Alessandria, così come vale a Roma. ${ }^{7}$ Dovunque è essenziale il ruolo della folla. Lo spettacolo del potere ha bisogno di trovare un'eco nella partecipazione del popolo. Si potrebbero citare svariati testi di poesia ellenistica: mi limito al XV Idillio di Teocrito, Le donne alla festa di Adone. Gorgo e Prassinoa attraversano la città per recarsi al palazzo del re, e dialogo e rappresentazione sono dominati dal motivo della grande quantità di persone che sono affluite. Uno dei tratti mimici più brillanti sta appunto nei piccoli incidenti, nei contatti fisici, nelle battute che vengono scambiate in questa enorme calca.

Questa poesia della folla è un elemento importante della elegia ovidiana, che si tratti della elegante moltitudine di donne a teatro (Ars 1.93-8), della turba cosmopolita della naumachia (1.175-6) o degli spettatori del trionfo (1.217). Il motivo perdura ancora nella produzione dell'esilio: a proposito del trionfo di Tiberio si dice, Pont. 2.1.21-4:

Indice te didici nuper visenda coisse innumeras gentes ad ducis ora sui, quaeque capit vastis inmensum moenibus orbem, hospitiis Romam vix habuisse locum.

Con la tua rivelazione ho appreso che poco tempo fa genti senza numero sono convenute per vedere il volto del loro condottiero,

e Roma che racchiude con le sue vaste mura il mondo immenso a stento ha avuto spazio per ospitarli.

Questa folla è la testimonianza della grandezza del principe e della sicurezza e prosperità che garantisce. Lo stesso motivo era risuonato nell'elogio di Tolemeo in Teocrito (15.44-6) e in Ovidio è volto ad esprimere le lodi di Augusto. Il poeta diviene uno tra i molti e questo è essenziale per esplicitare la sua organicità rispetto al regime imperiale. Essere parte della folla e cantare il principe: sembrerebbe semplice, ma in questi autori, sofisticati e callimachei, un simile obiettivo genera un atteggiamento complesso.

porta lo studioso a intendere legam come "leggerò", sviluppo dell'opposizione tra la storia di cui si è protagonisti e quella che semplicemente si legge sui libri.

${ }^{6}$ Difficile pensare che fata indichi la morte: così invece Hollis, 2007, p. 244-5.

${ }^{7}$ Sulla visibilità imperiale, e non solo, cf. Hekster, 2005, p. 158-76, in particolare p. 167-76; per l'età ellenistica utile Strootman, 2014, p. 42-53. 
Un ruolo importante nella proposizione del motivo è rivestito da Orazio. ${ }^{8}$ In Carm. 3.14 Herculis ritu modo dictus, o plebs, l'ode che canta il ritorno di Augusto dalla Spagna, ${ }^{9}$ il poeta assume la funzione di chi si rivolge al popolo. In apertura riudiamo l'apostrofe a mo' di

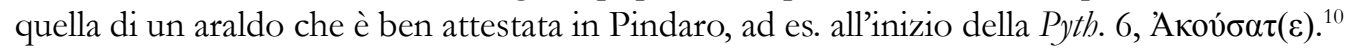

In Carm. 3.14 le prime tre strofe celebrano l'occasione pubblica con linguaggio religioso e patriottico. Prima di proclamare ciò che ci si attende che ciascuno faccia nell'occasione, Orazio si rivolge al popolo in un modo inconsueto per Roma. L'apostrofe $o$ plebs effettivamente suscita degli interrogativi e pare problematica. Eduard Fraenke ${ }^{11}$ prova a chiarirla, sulla base di uno studio di Jacob Wackernagel, ${ }^{12}$ con l'uso nelle apostrofi del nominativo di $\lambda \alpha o ́ \varsigma$ e $\lambda \varepsilon \omega ́ \varsigma$ invece del vocativo nella tragedia greca; gli unici paralleli sono in Livio, 1.24.7: audi tu, populus Albanus, "ascolta tu, popolo Albano", e Ovidio, Fast. 4.731: $i$, pete virginea, populus, suffimen ab ara, "va', o popolo, prendi dall'ara virginea i materiali da far fumigare". ${ }^{13}$

La prima parte del componimento oraziano è molto formale, ma con un'enfasi sull'aspetto privato: si dice dei Penati della casa di Augusto (v. 3), della gioia di Livia per suo marito (v. 5), dell'affetto di Ottavia per suo fratello (v. 7), della grazia e riverenza delle matrone (vv. 7-8), del silenzio imposto ai ragazzi e alle ragazze (vv. 10-2):

Herculis ritu modo dictus, o plebs,

morte venalem petiisse laurum

Caesar Hispana repetit Penatis

victor ab ora.

Unico gaudens mulier marito

prodeat iustis operata divis

et soror clari ducis et decorae

supplice vitta

virginum matres iuvenumque nuper

sospitum; vos, o pueri et puellae

\footnotetext{
${ }^{8}$ Per questa ode e quelle che seguono si pone il problema dell'attribuzione di un ruolo privato o pubblico (e in quale misura). Orazio si confronta per questo con la tradizione poetica greca. La questione è presentata efficacemente da Carey, 2016, p. 187-9 su 3.14.

${ }^{9}$ È messa opportunamente in parallelo con l'epigramma di Gallo per la partecipazione del privato cittadino alle vittorie del princeps: Barchiesi, 1981, p. 158; il confronto è sviluppato in Lyne, 1995, p. 170-1; vd. anche Putnam, 1986, p. 57-60.

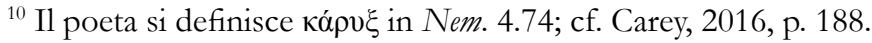

${ }^{11}$ Fraenkel, 1957, p. 289 n. 1.

${ }^{12}$ Wackernagel, 1912, p. 15-6 (1955, II, p. 982-3).

${ }^{13}$ Questo verso introduce le istruzioni che Ovidio, in qualità di autore didascalico, impartisce per lo svolgimento della festa di Pale. La dea gli esprime il suo favore ed egli riceve una consacrazione che gli conferisce l'autorità necessaria (v. 721-30). Al di là di questo inizio, il passo non si sviluppa nella direzione della mimesi della cerimonia, descritta mentre si sta svolgendo.
} 
iam virum expertae, ${ }^{14}$ male nominatis ${ }^{15}$

parcite verbis.

Al modo di Ercole, Cesare, si diceva, or ora era andato a raccogliere allori

a prezzo della vita e adesso torna, o plebe, vittorioso

alla sua dimora dalla terra di Spagna.

Gli vada incontro, offerti i sacrifici ai giusti

dèi, la sposa orgogliosa di quell'uomo

unico, e la sorella dell'illustre

condottiero, e, adorne della supplice benda,

le madri delle fanciulle e dei giovani

che ritornano salvi. E voi, ragazzi

e fanciulle già esperte di amori, astenetevi da parole infauste.

Dare le istruzioni a ciascuno è un tratto di tecnica callimachea, che ci è ben attestato negli Inni (2, 5 e 6$)$. Il poeta annuncia ciò che ci si attende che ciascuno faccia. A titolo puramente esemplificativo valga l'inizio dell'Inno a Pallade:

Voi che versate il lavacro di Pallade, uscite tutte, uscite: or ora il nitrito delle cavalle sacre ho udito, e la dea è pronta a venire.

Accorrete, o bionde Pelasghe, accorrete.

(Traduzione di G. B. D’Alessio)

La mimesi, l'imitazione dell'andamento della festa nel suo sviluppo, in questo caso e negli altri, è un elemento tipico della poesia ellenistica, ormai lontana dalla realtà concreta del rito, ancorché di tali testi non si possa escludere una fruizione puramente aurale. ${ }^{16}$

In Orazio segue una strofa che collega la parte pubblica con quella privata (mihi festus).

\footnotetext{
${ }^{14}$ Il testo pare qui effettivamente problematico in quanto dà il senso opposto a quello che ci attenderemmo: utile discussione in Nisbet, 1983, p. 112-4. Nisbet; Rudd, 2004, p. 185-6, pongono le cruces ed effettivamente sembrerebbe molto difficile superare la polarità dell'espressione, dove i pueri non pare che possano essere su un piano differente da quello delle puellae: identificare i pueri con gli iuvenes non si configura agevole, pace Syndikus, 1990, p. 147-8, n. 30.

${ }^{15}$ Non è semplice, inoltre, spiegare male nominatis con il valore di "infausto", che tuttavia si può ricavare

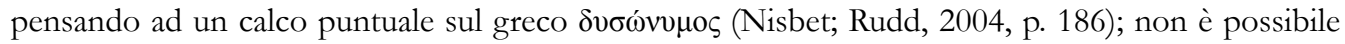
accettare lo iato male ominatis (anche pensando che i due termini possano essere sentiti fusi in un'unica parola) che ci è trasmesso da una parte della tradizione.

${ }^{16}$ Sintesi della questione e bibliografia in D'Alessio, 1996, v. I, p. 12-3, a cui si può aggiungere la messa a punto di Fantuzzi; Hunter, 2002, p. 33-5. La complessità della "mimesi" negli Inni è affrontata efficacemente da Depew, 1993, p. 57-77.
} 
Hic dies vere mihi festus atras eximet curas; ego nec tumultum nec mori per vim metuam tenente

Caesare terras.

Davvero questo giorno di festa scaccerà da me gli oscuri affanni. Non temerò tumulti né morte violenta, perché Cesare governa la terra.

Seguono poi tre strofe in cui si dice dei preparativi del simposio e alla fine la voce personale del singolo è perfettamente individuata. A questo punto è evidente che l'insieme dell'evento, presentato con tale enargeia, è soltanto immaginato, e che il poeta va collocato nella sua dimensione privata. ${ }^{17}$

Significativamente la medesima struttura è presente nella elegia 6 del IV libro di Properzio, il grande componimento (molto sperimentale) che canta la battaglia di Azio in distici elegiaci. L'inizio, con l'esplicita menzione di Filita e di Callimaco, è costituito da un invito a celebrare il rito:

Sacra facit vates: sint ora faventia sacris, et cadat ante meos icta iuvenca focos.

serta Philiteis certet Romana corymbis, et Cyrenaeas urna ministret aquas.

costum molle date et blandi mihi turis honores, terque focum circa laneus orbis eat.

Il vate celebra un rito: le voci assecondino il sacrificio, e una giovenca cada immolata davanti ai miei fuochi.

La corona romana gareggi con i corimbi di Filita e l'urna versi acque di Cirene.

Datemi i molli profumi del costo e l'onore del gradito incenso, $\quad 5$ e tre volte la benda di lana giri intorno all'altare.

Segue la narrazione encomiastica dell'evento. A un certo punto però Properzio dice di aver lasciato abbastanza spazio al canto dell'impresa del princeps:

\footnotetext{
${ }^{17}$ Albert, 1988, p. 142. Questo ha anche la funzione di garantire la 'sincerità' dell'elogio: cf. Lyne, 1995, p. 171. L'ode è, naturalmente, complessa, e ad essa dà spessore la storia, anche personale di Orazio, dalla quale sembra eccessivo ricavare sentimenti attuali di opposizione nei confronti di Augusto (queste posizioni sono raccolte in Cremona, 1982, p. 327-30, che peraltro se ne discosta). L'unità del componimento e il suo tono sono ben presentati da Syndikus, 1990, p. 142-53.
} 
bella satis cecini: citharam iam poscit Apollo victor et ad placidos exuit arma choros.

candida nunc molli subeant convivia luco; blanditiaeque fluant per mea colla rosae, vinaque fundantur prelis elisa Falernis, terque lavet nostras spica Cilissa comas.

Ho cantato abbastanza le guerre: Apollo vincitore ormai chiede la cetra, e si spoglia delle armi per i placidi cori.

Ora si celebrino conviti in candida veste in un ameno boschetto, le carezze delle rose scorrano lungo il mio collo, si versino i vini premuti dai torchi di Falerno, e la spiga cilicia unga le nostre chiome.

Propriamente è Apollo che, dopo aver determinato la vittoria di Ottaviano, intende darsi al canto e induce il poeta al simposio, e questa è dunque la collocazione del componimento di Properzio, come di quello di Orazio - nonostante l'apostrofe o plebs, che evidentemente era solo formale. Un paragone interessante lo si può avere con l'epodo 16: Orazio in quel caso, con la sua proposta di lasciare Roma, pare che parli ad una assemblea; $\mathrm{i}$ modi però non sono quelli del senato di Roma, ma piuttosto quelli in uso in una polis greca. Orazio si rivolge al gruppo ristretto di suoi compagni assumendo il ruolo fittizio di un araldo greco, che convoca l'assemblea dei cittadini e ai pii trasmette la volontà degli dèi, della quale si fa mediatore in quanto profeta e poeta. ${ }^{18}$

Quanto alla celebrazione, anche per la sua collocazione nel simposio ci sono precedenti nella poesia ellenistica: è stato individuato un parallelo con la Pannychis, la Festa notturna di Callimaco, di cui purtroppo ci è rimasto un unico frammento, oltre all'argomento che recita: "Canto simposiale per i Dioscuri. Canta anche Elena e prega che accettino il sacrificio. È anche un'esortazione ai convitati a rimanere svegli." Quanto al frammento (227 Pfeiffer),

Apollo è nel coro: odo il suono della lira.

E sento presenti gli Amori: c'è anche Afrodite,

La situazione, descritta in toni che richiamano l'inizio mimetico dell'Inno ad Apollo e di altri Inni, introduce un'epifania della divinità che qui non implica che si tratti di un rito in suo onore, né che vi sia una qualche sorta di mascherata. La presenza di Apollo è rivelata dalla musica e dalle danze: quella di Afrodite e degli Amori dall'atmosfera conviviale. Abbiamo dunque un simposio ufficiale, come si deduce dalla menzione delle divinità legate in particolare modo ai Tolemei, i Dioscuri ed Elena.

Nel III libro delle Odi la celebrazione è pertanto mediata.

Nel libro IV delle Odi si possono individuare cambiamenti significativi.

\footnotetext{
${ }^{18}$ Sintesi in Watson, 2003, p. 487; la stessa osservazione vale per il contesto dell'epodo 7, con l'apostrofe ai scelesti, che non esclude affatto un'ambientazione simposiaca.
} 
Nel secondo componimento, dopo una complessa riflessione, rivolta a Iullo Antonio, sulla difficoltà di imitare la poesia di Pindaro, il poeta esibisce la sua partecipazione ai canti della folla:

Tum meae, si quid loquar audiendum, vocis accedet bona pars, et: "O sol pulcher, o laudande!” canam recepto

Caesare felix;

teque, dum procedis, io Triumphe!

non semel dicemus, io Triumphe!

civitas omnis, dabimusque divis

tura benignis.

Allora, se dirò qualcosa

degna di ascolto, si aggiungerà

con forza la mia voce, e «Sole radioso, a te gloria!», dirò felice del ritorno

di Cesare, e al tuo incedere «Evviva Trionfo!»

non una sola volta diremo, «Evviva Trionfo!»

noi tutti cittadini e offriremo incenso

agli dèi benevoli.

Nelle due ultime strofe Orazio aggiunge che Iullo potrà offrire in ringraziamento il sontuoso sacrificio di dieci tori e dieci mucche, laddove l'offerta del poeta si limiterà ad un vitello.

A Iullo viene detto che l'imitazione della poesia di Pindaro presenta difficoltà e rischi: chi la tenta può finire come Icaro. Orazio sceglie per sé un ruolo differente, quello del poeta callimacheo (v. 27-32)

ego apis Matinae

more modoque

grata carpentis thyma per laborem

plurimum circa nemus uvidique

Tiburis ripas operosa parvus

carmina fingo.

con gli usi ed il modo

dell'ape matina,

suggendo con un lungo travaglio dolce

timo, intorno ai boschi e alle umide

ripe di Tivoli, io, modesto,

compongo laboriosi carmi. 
Iullo Antonio, dotato com'è di una maggiore capacità poetica, potrà celebrare epicamente $^{19}$ il trionfo di Augusto, che è il dono più bello concesso alla terra dal fato e dagli dèi e cantare la gioia della città in festa per il suo ritorno.

Il componimento è molto complesso e se semplifichiamo all'estremo ne possiamo riassumere in questo modo lo sviluppo: il ritorno che sembra vicino di Augusto dalla Gallia impone ai letterati di corte il problema di celebrare l'evento. Orazio sa bene che per farlo in maniera adeguata sarebbe necessario un nuovo Pindaro, che però è ineguagliabile, sia nel lessico che negli schemi metrici, che nelle doti naturali. ${ }^{20}$ Orazio sarebbe il poeta più adatto, tanto più che Carm. 4.4 e 14 sono odi per molti aspetti compiutamente pindariche - e uno dei problemi del rapporto di Orazio con Pindaro sta nel fatto che il poeta latino mostra di essere pari a quello greco; e allora, dove sta l'impossibilità? Orazio vuole ritagliarsi un luogo suo proprio (forse in questo sta l'accentuazione dell'arduo compito) e perciò ripropone la sua scelta del piccolo, dello spazio protetto, l'angulus. Iullo Antonio può invece celebrare il principe con la poesia epica di cui ha dato dimostrazione di essere capace. Quello di Orazio sarà un atto di omaggio che da un lato non gli impedirà di offrire in modo accortamente allusivo e privilegiando toni modesti e popolari ${ }^{21}$ il suo canto di elogio al principe, dall'altro gli consentirà di sviluppare quel discorso di poetica, per cui potrà rivendicare a sé il culto di una poesia raffinata e frutto di intenso labor limae, nel pieno rispetto dei canoni della poesia alessandrina che nei confronti delle posizioni teoriche di Pindaro avevano un debito importante.

Un'altra spiegazione interessante della difficoltà di imitare Pindaro è stata vista opportunamente nell'impossibilità, della quale Orazio era ben consapevole, di ricostruire il contesto della performance proprio dei componimenti corali, ${ }^{22}$ che non sono soltanto epinici, ma anche ditirambi - e in questo senso è notevole il fatto che l'ode di Bacco, 3.25, contempli un momento di elogio per Cesare, molto in evidenza: "In quali antri sarò udito alzare / l'eterna gloria dell'eccelso Cesare / inserendolo fra le stelle e nel concilio di Giove?" (vv. 3-6). ${ }^{23} \mathrm{Vi}$ sono però punti in cui Pindaro riferisce ciò che dice la gente, anche se propriamente si tratta di un gruppo di synetoi:

\footnotetext{
${ }^{19}$ Harrison, 1995 , p. $115-22$.

${ }^{20} \mathrm{Il}$ modo in cui questo problema coinvolga quanto era presente negli antichi commenti a Pindaro è messo bene in evidenza da Bitto, 2012, p. 412-3, che a p. 420 n. 233 sottolinea inoltre come sia importante la sequenza dei componimenti del libro IV, secondo la quale l'ode con la dichiarazione di incapacità, che produce il canto non-pindarico dei v. 46-7, effettivamente precede quelle che rappresentano un tentativo di pindarismo.

${ }^{21}$ Questi vengono esibiti nella parte finale del componimento con una densità di espressioni informali ai v. 45-52: cf. Di Liddo, 2004, p. 60-4.

22 Athanassaki, 2016, p. 134. Un elemento pindarico importante che non ha corrispondenza nella lirica oraziana è naturalmente la metrica: Barchiesi, 2009, p. 332.

${ }^{23} \mathrm{Il}$ modo in cui l'ispirazione bacchica si combina con quella apollinea, e quella pindarica con quella callimachea, è illustrato efficacemente da Schiesaro, 2009, p. 61-79.
} 
Tale è l'uomo che lodano i saggi;

io dirò quello che dicono:

egli nutre intelletto e parola

maggiore degli anni ... (Pyth. 5.107-11).

Il ruolo di chi parla, che si presenta inserito all'interno di un gruppo, può essere stato utile spunto per Orazio. ${ }^{24}$

Secondo i tratti caratteristici della recusatio, il poeta propone ciò che non canta, nella fattispecie la descrizione del corteo trionfale, che spetterebbe a Iullo. Le parole che lui invece pronuncia, o sol pulcher, o laudande, corrispondono stilisticamente e metricamente alla prima metà di un versus quadratus, cioè di quel settenario trocaico che era il metro tipico dei carmina triumphalia, intonati sin dal tempo di Camillo dai soldati o dalla folla presente al trionfo, e quindi di chiaro sapore popolare. ${ }^{25} \mathrm{Il}$ poeta pertanto si unisce alla folla e vuole esserne parte. Questo accade, però, suo modo. L'emistichio è spezzato ed è inserito in un contesto metrico differente. L'immagine di Augusto come sole ${ }^{26}$ - sembra questa l'interpretazione più probabile, di contro all'ipotesi di rinvenire un'invocazione al giorno, al dies, evidentemente felice - riveste una funzione letterariamente importante, in quanto costituisce un momento di contatto e un rinvio all'ode 5 del medesimo libro.

In questo componimento colpisce senz'altro la ricchezza dei motivi propagandistici e dei topoi encomiastici, primo fra tutti, appunto, quello dell'assimilazione dell'imperatore al sole, che permette un accostamento ai basilikà mele ellenistici. È come se, nella sua complessità, avesse l'obiettivo di esprimere attese soteriologiche diffuse a un livello generale, anche attraverso forme popolareggianti di elogio del princeps. Si tratta di un frutto molto particolare della produzione oraziana. Sembra che il poeta voglia offrirci sul sovrano lo sguardo di un uomo del popolo: la patria desidera il ritorno di Augusto e vengono espressi i sentimenti, diremmo, del cittadino medio. Il personaggio Orazio compare soltanto alla fine, significativamente nel contesto del simposio. ${ }^{27}$ Subito prima (v. 31-6) si è detto delle molte preghiere e libagioni del contadino ad Augusto; a queste si unisce Orazio (Carm. 4.5.36-40):

$$
\begin{aligned}
& \text { "Longas o utinam, dux bone, ferias } \\
& \text { praestes Hesperiae!" dicimus integro } \\
& \text { sicco mane die, dicimus uvidi, } \\
& \text { cum sol Oceano subest. }
\end{aligned}
$$

\footnotetext{
${ }^{24}$ Athanassaki, 2016, p. 139.

${ }^{25}$ Così R. Heinze nella nota a Carm. 4.2.46 (Berlin: Weidmann, 1930, p. 397).

${ }^{26}$ Per Augusto, sol e Apollo nel contesto del trionfo di Azio cf. Hardie, 1986, p. 116.

${ }^{27}$ Anche caratteri come questi hanno fatto ipotizzare per questa ode una performance pubblica: Du Quesnay, 1995, p. 143-8; contra Fedeli; Ciccarelli, 2008, p. 261. Proporre un'esecuzione pubblica implica naturalmente un'interpretazione di questi componimenti come espressione di un'ideologia ufficiale, laddove immaginare una diffusione puramente scritta restituisce una maggiore complessità.
} 
"O buon condottiero, possa tu

donare all'Esperia lunghi giorni di festa!’, diciamo

sobri sul far del giorno, e diciamo

ebbri quando il sole scompare nell'Oceano.

Di nuovo, come nella sesta elegia del IV libro di Properzio, dopo la celebrazione il poeta si ritira nel simposio che è però luogo in cui trova espressione, nei modi appropriati, la fedeltà ad Augusto.

L'Ode 4.5 è molto notevole, singolarmente semplice, anche da un punto di vista della struttura sintattica, che si potrebbe definire quasi epigrafica: solo nell'epodo 2 si ha una simile sequenza di frasi piane coincidenti sintatticamente con il verso come qui ai vv. 17-24. Basti l'esempio solo di una strofa (vv. 17-20):

Tutus bos etenim rura perambulat, nutrit rura Ceres almaque Faustitas, pacatum volitant per mare navitae, culpari metuit fides.

Infatti il bue percorre sicuro i campi, i campi li nutrono Cerere e la feconda Prosperità; i marinai navigano su acque pacificate; la Lealtà non accetta macchia.

Sembra davvero l'espressione dei sentimenti dell'uomo comune. L'ordinata successione dei versi collegati per asindeto e caratterizzati dall'alternanza di dati concreti e concetti astratti personificati, non solo fa pensare ad una serie quasi infinita di benefici morali e materiali, ma sembra anche riprodurre immediatamente la tranquillità e la sicurezza offerte al popolo romano dal regime. La successione di frasi collegate per asindeto, va detto, è tipica delle aretalogie inniche; nel carme oraziano, poi, all'impiego di tale tecnica si associa quello di toni e stilemi caratteristici della teofania: un'utile esemplificazione può venire dalla sezione dell'Inno ad Artemide di Callimaco, in cui si dice dei benefíci prodotti dalla benevolenza della dea: "A chi invece tu volgi sorridente e propizia lo sguardo, / a quelli il campo è ricco di spighe, è ricca la stirpe / dei quadrupedi e la casa si accresce ..." (v. 129-31; traduzione di G. B. D’Alessio), motivi adattati anche nella poesia panegiristica, ad es. Theocr. 17.95$114 .{ }^{28}$ Orazio celebra il buon governo di Augusto con l'impiego sia di espedienti stilistici caratteristici dell'inno, sia di motivi più specificamente politici ed etici.

Naturalmente Orazio è sempre un poeta complesso. Consideriamo i primi versi:

Divis orte bonis, optume Romulae custos gentis, abes iam nimium diu; maturum reditum pollicitus patrum sancto consilio redi.

Lucem redde tuae, dux bone, patriae...

\footnotetext{
${ }^{28}$ Fedeli; Ciccarelli, 2008, p. 277. Per Teocrito utile Hunter, 2003, p. 170-84.
} 
O prole di benevoli iddii, ottimo custode della romulea gente, già da troppo tempo ci sei lontano. Promesso un sollecito ritorno al sacro consiglio dei padri, ritorna!

Rendi, o buon condottiero, la luce alla tua patria ... Skutsch: ${ }^{29}$

In questi nessi che paiono così formulari si nota però la ripresa di Ennio, ann. 105-9

Pectora ... tenet desiderium, simul inter sese sic memorant: "o Romule, Romule die, qualem te patriae custodem di genuerunt! O pater, o genitor, o sanguen dis oriundum, tu produxisti nos intra luminis oras".

I cuori li stringe il rimpianto, e tra sé così ripetono: "O Romolo, divino Romolo, quale custode alla patria ti generarono gli dèi! O padre, o genitore, o sangue nato dagli dèi, tu ci hai portato al mondo della luce."

Nel carme di Orazio individuiamo tracce di questo passo: v. 1 divis orte, Romulae, 5 lucem, patriae; in seguito vi è la similitudine tra la patria e la madre che sente la mancanza del giovane figlio lontano da un anno. ${ }^{30}$ Come nella rassegna dei grandi Romani del VI libro dell'Eneide Augusto segue immediatamente Romolo, così qui Orazio stabilisce un implicito parallelo tra i due fondatori di Roma e si colloca nella dimensione della poesia encomiastica enniana, la poesia che, come viene esplicitamente argomentato nell'Ode 8 , è destinata a durare più a lungo di ogni altra creazione dell'uomo.

Segnalo subito un passo ovidiano dalla poesia dell'esilio, del tutto analogo a questi oraziani, Tr. 2.57-60:

Optavi, peteres caelestia sidera tarde,

parsque fui turbae parva precantis idem, et pia tura dedi pro te, cumque omnibus unus

ipse quoque adiuvi publica vota meis.

Ho desiderato che tu ti dirigessi tardi verso le stelle del cielo

e sono stato piccola parte della folla che levava questa preghiera, e ho offerto per te piamente l'incenso e assieme a tutti

ho aggiunto i miei voti a quelli del popolo.

\footnotetext{
${ }^{29}$ Fraenkel, 1957, p. 441-2.

${ }^{30}$ Su questa e altre analogie cf. Putnam, 1986, p. 103-7.
} 
Ovidio, da Tomi, ha l'assoluta necessità di evidenziare il suo lealismo, la sua fedeltà all'imperatore, e lo fa inserendosi nella folla che offre preghiere e suppliche. ${ }^{31}$ In Tr. 4.2 .16 (cf. infra) Ovidio dice del posto vuoto che ha lasciato nella folla di Roma che rivolge invocazioni in favore dell'imperatore.

Sarebbe però forse interessante affrontare il modo in cui questo tema si inserisce nella rappresentazione delle pubbliche cerimonie, e così si torna al punto da cui ha preso le mosse il discorso, la collocazione del poeta in occasione di un trionfo. I precedenti negli altri autori elegiaci sono naturalmente molto importanti. Il trionfo è il culmine della celebrazione del sovrano vittorioso, che con le sue conquiste garantisce la sua natura di vincitore predestinato dagli dèi. Basti pensare anche solo alla descrizione epica della cerimonia in Virgilio, Aen. 8.714-28: nella sua accentuata dimensione religiosa, posta com'è di seguito al quadro della battaglia di Azio, costituisce una sorta di punto di arrivo della storia romana. Vi si sottolinea la pietas del principe, e il dettagliato catalogo dei popoli sottomessi, che pur non riproduce con esattezza quello della realtà storica, vale ad esprimere il compimento del destino imperiale. A questo fine sono funzionalizzate anche le componenti pittoresche nella descrizione delle varie genti e nelle personificazioni dei fiumi, che entrano a far parte delle ekphraseis successive.

In Tibullo il trionfo è un elemento importante in 1.7, elegia dedicata a Messalla Corvino, di cui è opportuno mettere in rilievo la novità. Anche senza giungere a definirla un 'epinicio' (sembra piuttosto una sintesi di vari generi), rimane evidente il suo carattere sperimentale. ${ }^{32}$ Suo obiettivo principale è l'encomio dell'amico potente e proprio per questo aspetto vi possono essere somiglianze con alcuni caratteri dell'elegia ovidiana dell'esilio, che si concentra anch'essa sulle lodi per singoli personaggi di rilievo. Attraverso lo strumento della profezia, la vittoria sugli Aquitani è fatta coincidere con il compleanno di Messalla, in un intreccio di motivi che producono una lode complessa. Garanzia di veridicità del vaticinio è appunto la celebrazione del trionfo, colto nei due quadri dei nemici incatenati e del condottiero romano sul carro (vv. 5-8). In tale contesto un caso notevole è la conclusione di Tibullo 2.5, con la profezia per Messalino (vv. 115-8), da accostare alla sezione su Germanico di Pont. 2.1: $:^{33}$ sono encomi rivolti a giovani inseriti in una ben precisa tradizione familiare,

\footnotetext{
${ }^{31}$ Poco dopo Ovidio mette in rilievo di essere stato parte della sua patria, con una formulazione analoga: v. 157-8 perpatriam, quae te tuta et secura parente est, / cuius, ut in populo, pars ego nuper eram.

${ }^{32}$ Murgatroyd, 2001 [1980], p. 208-13. La novità di un epinicio in distici per fatti bellici, che Murgatroyd evidenzia (p. 210-1), si riduce se si pensa ad analoghi componimenti dell'Oriente greco. Ci è giunto un frammento encomiastico a proposito di vittorie in una campagna militare vera e propria, SH 969, il cui senso generale sembra essere: spesso giunse in Egitto la fama delle tue vittorie (v. 1-2); con azioni eroiche ti sei guadagnato una gloria che resterà nei libri (v. 3-4). Prima infatti quando infuriavano (i Galati) minacciando l'Egitto (vv. 5-6), si verificarono stragi (v. 7-8): cf. Barbantani, 2001, p. 73-116. Sono stati anche segnalati punti di contatto con gli epinici di Callimaco: Bulloch, 1973, p. 80, con ulteriori rinvii.

33 Anche nel caso di Tibullo la possibilità di celebrazione è legata dalla salvezza del poeta, che qui dipende dalla donna da lui amata (v. 114 praemoneo, vati parce, puella, sacro).
} 
che si apprestano ad intraprendere la carriera che spetta di diritto ad un aristocratico nel primo caso, ad un principe nel secondo. Manca il senso della rottura con il mondo ufficiale.

Ciò si riscontra in Properzio. Vi sono apparizioni isolate del motivo, in contesti in cui è un semplice sinonimo di vita militare, rispetto alla quale il poeta manifesta un atteggiamento di indubbia repulsione; tuttavia nelle riprese più ampie, e. g. in Elegie 3.4, come abbiamo visto, non c'è una reale contestazione, ma semplicemente un'affermazione di alterità: il trionfo fa parte di un mondo in cui Properzio non vuole entrare. Ciò non toglie che in quest'elegia, addirittura, egli possa investirsi di una funzione di vates e di angur acquisendo, sia pure per un attimo soltanto, un ruolo ufficiale. In Elegie 3.1 si ha invece un esempio di come il trionfo possa venir assunto nel mondo elegiaco attraverso la complessa procedura per cui le varie funzioni dell'àmbito pubblico vengono assorbite e transcodificate. Il poeta elegiaco si autocelebra come se fosse un grande condottiero militare. Di fatto analoga è l'operazione di Ovidio in Am.1.2, benché lì si presenti come uno dei prigionieri nel corteo trionfale. Si tratta già di un esempio di quella che è stata chiamata "retorica congiuntiva": l'argomento serio non viene ridicolizzato, ma proposto in una visione della città e del regime che non è di opposizione e contestazione, anche se ne sviluppa alcuni caratteri in maniera univoca. ${ }^{34}$ Interessante il caso di Ars 1.171-228: Ovidio affronta il tema per dire che anche le manifestazioni ufficiali della città sono occasioni di facili incontri amorosi. Il trionfo è quello che Ovidio preannuncia come auspicio di felice conclusione dell'imminente campagna orientale di Gaio Cesare, nipote e figlio adottivo di Augusto. Lo spazio più ampio viene concesso alla pubblica festosità, ma non per questo bisogna sminuire $i$ tratti autenticamente celebrativi, sospettando in essi soltanto ipocrisia e ironica finzione. Nell'opera dell'esilio sono ripresi questi caratteri essenziali della poesia precedente, legati al tema della città e dello splendore che le viene dalla dinastia, come risulta più evidente da Pont. 2.1, piuttosto che da Tr. 4.2, anche se manca, rispetto all'Ars, un'atmosfera specificamente lieve e giocosa. Attraverso la sua passata esperienza poetica Ovidio si propone nel ruolo di panegirista "ufficiale", proprio per dimostrare una reale continuità. Vorrebbe inoltre suggerire che, pur essendo per il momento frustrato nelle sue aspirazioni, può e potrà dare molto dopo il suo ritorno; nel frattempo fornisce brevi saggi della poesia che realizzerebbe se gli venisse tolta la condanna. L'aspetto scenografico, come per ogni poeta d'occasione che si rispetti, ha perciò un ruolo di rilievo, così come, in Pont. 2.1, l'uso del discorso indiretto (sarebbe la fama a parlare) per la descrizione della cerimonia, da cui deriva l'impressione di un accentuato distacco. In quest'ottica è importante sottolineare la funzione del finale, in cui il canto è legato alla salvezza del poeta.

In confronto a Tr. 4.2, elegia ancora vicina ai modi dell' Ars, elemento distintivo di Pont. 2.1 è la connessione con un celebrando preciso: è indubbio che molto dalla sezione iniziale si riverberi anche, e soprattutto, sulla profezia rivolta a Germanico, tanto che alla fine del componimento il trionfo di Tiberio potrebbe apparirci perfino relegato in secondo piano. Nelle Ex Ponto quest'elegia è il primo passo compiuto da Ovidio nei confronti del giovane principe e, in quanto tale, è collocata in una posizione di spicco ed ha caratteristiche

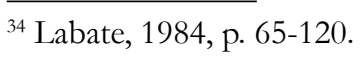


proprie. La gerarchia in senso dinastico riceve tuttavia debita attenzione con la sottolineatura del rapporto di filiazione tra Tiberio e Germanico, quasi si trattasse di una progenitura diretta (in modo analogo a quello in cui è presentata la parentela tra Cesare e il suo erede nel finale delle Metamorfosi), mentre la figura di Augusto, sullo sfondo, assurge già ad una grandezza sovrannaturale.

Oltre alle lodi del princeps e della famiglia dominante, Ovidio produce una poesia encomiastica anche in riferimento a importanti figure di nobiles, i cui risultati più evidenti si rinvengono nel libro IV delle Ex Ponto, a proposito in particolare delle celebrazioni per il consolato (4, Sesto Pompeo; 9, Grecino), che in entrambi i casi si proietta nel futuro oppure (5) trova espressione attraverso l'apostrofe agli elegi. Ovidio aspira a essere uno dei tanti partecipanti e mette in rilievo come anche lui possa essere toccato da ciò che piace alla massa. Questo non si realizza ormai nei trionfi, ma nella cerimonia di inizio consolato che riguarda i potenti amici dell'esule. In Pont. 4.4.27-8 è in evidenza proprio la grandezza della folla:

Cernere iam videor rumpi paene atria turba et populum laedi deficiente loco.

Già mi sembra di vedere che per poco gli atri non sono sfondati dalla folla e il popolo è schiacciato per la mancanza di spazio.

Ovidio attua il procedimento della visione mentale riferita ad un evento futuro: in questo modo la poesia celebrativa che egli intende produrre potrà rivelarsi all'altezza della situazione, proprio perché egli trova in se stesso la possibilità di realizzarla. In Pont. 3.4 il componimento autonomo sul trionfo di Tiberio ha bisogno dell'indulgenza del pubblico, laddove la profezia rivolta a Livia per quello futuro di Germanico ha un tono davvero ispirato che suona adeguato al compito che $\mathrm{a}$ Ovidio si pone. Le risorse a questo fine le ricaverà dalla sua stessa condizione di poeta che, come sappiamo dal finale delle Metamorfosi, è in grado di predire il futuro. Le parole della fama producono poi una visione mentale molto efficace, che quasi simboleggia la capacità di enargeia dell'autore. Paralleli significativi si possono rinvenire in due testi: la descrizione della potente visione mentale che è suscitata dalle parole di Epicuro all'inizio del III libro di Lucrezio (vv. 14-30), e l'esortazione rivolta al proprio animo da Properzio 2.10.11-8, che lo invita a raggiungere un livello superiore e a cantare le vittorie di Augusto, e in tre distici ci offre effettivamente un esempio di questa poesia.

Oltre a 4.4, anche 4.5 è dedicata all'inizio di consolato, come poi 4.9. Nella prima metà di 4.9 lo sviluppo della celebrazione del nuovo console riunisce entrambe le tematiche delle due elegie per Sesto Pompeo: nel primo testo si insisteva sulla cerimonia di inaugurazione del consolato, di cui qui sono ripresi svariati elementi, con una grande evidenza rivolta ai particolari descrittivi, che sono visti, potremmo dire, dalla prospettiva del popolo. Da ciò l'accentuazione dei tratti di sontuosità e solennità, in armonia con quella partecipe rappresentazione della vita cittadina che fin dagli Amores, abbiamo visto, è uno dei caratteri principali dell'opera ovidiana. In Pont. 4.5, invece, si aveva, come qui ai vv. 41-54, la descrizione dell'attività consueta di un console, che in ambedue i casi viene riferita per interposta persona, nella elegia più antica attraverso gli elegi, qui per mezzo della visione della 
mente. La riproposizione del medesimo tema consente all'esule di offrire una tipologia dei componimenti che potrebbero far parte del suo prontuario al ritorno dall'esilio, collocabili sotto l'etichetta "celebrazione dell'inizio consolato"; in prospettiva, poi, dato che questa carica viene rivestita da una sequenza di esponenti della nobilitas, il poeta potrebbe dare vita ad una galleria di Romani che contano, come in fondo fa Orazio nel IV libro delle Odi.

Nel caso di Sesto Pompeo c'è il rimpianto per non poter essere presente; in quello di Grecino, in Pont. 4.9, la descrizione del poeta nella folla invece viene sviluppata con una certa ampiezza, per effetto della visione mentale (vv. 15-26):

Illa, confiteor, sic essem luce superbus ut caperet fastus vix domus ulla meos, dumque latus sancti cingit tibi turba senatus, consulis ante pedes ire iuberer eques

et, quamquam cuperem semper tibi proximus esse, gauderem lateris non habuisse locum

nec querulus, turba quamvis eliderer, essem, sed foret a populo tum mihi dulce premi.

Prospicerem gaudens quantus foret agminis ordo densaque quam longum turba teneret iter, quoque magis noris quam me vulgaria tangant, spectarem qualis purpura te tegeret.

Quel giorno, lo confesso, mi sentirei così superbo che a stento una casa riuscirebbe a contenere il mio orgoglio; e mentre la folla del sacro senato ti attornierebbe, io, cavaliere, sarei invitato a procedere davanti al console; e pur desiderando esserti sempre il più vicino gioirei di non avere posto al tuo fianco; e non starei a lamentarmi, benché schiacciato dalla ressa, ma sarebbe per me un piacere essere stretto dalla gente.

Guarderei con gioia la grandezza del corteo e la lunghezza dell'itinerario occupato da una folla fitta.

E perché tu sappia bene quanto mi affascinino anche le cose comuni, 25 guarderei la qualità della porpora che ti rivestisse.

Properzio si teneva a margine dell'evento; Orazio, prima all'esterno, poi entra a far parte della moltitudine e insieme ad essa eleva il suo canto, con un significativo cambiamento dalla prima raccolta delle Odi al libro IV. Orazio però può negoziare per sé una posizione particolare e tutto il IV libro rappresenta una riflessione sulla produzione di poesia celebrativa. Ovidio compie un itinerario complesso che va dalla rappresentazione del trionfo come occasione di incontri galanti, alla volontà di entrare nella folla della grande città, che assomiglia così tanto ad Alessandria e che lui tanto ora desidera. Poter essere un elemento, una cellula della folla che la riempie viene ad essere un desiderio davvero vivo, che per lui segnerebbe il massimo dell'integrazione ora che è exclusus. 


\section{RIfERIMENTI}

ALBERT, Winfried. Das mimetische Gedicht in der Antike. Frankfurt a. M.: Athenäum, 1988.

ATHANASSAKI, Lucia. Pindarum quisquis studet aemulari: Greek and Roman civic performance contexts (Pindar's Fourth and Fifth Pythians and Horace's Odes 4.2). In: DELIGNON, Bénédicte; LE MEUR, Nadine; THÉVENAZ, Olivier (Ed.). La poésie lyrique dans la cité antique. Les Odes d'Horace au miroir de la lyrique grecque archaïque. Paris: Librairie de Boccard, 2016. p. 131-58. (Collection du CEROR).

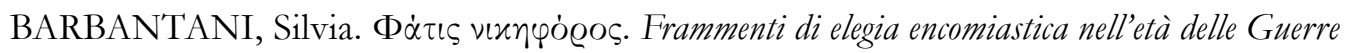
Galatiche: Supplementum Hellenisticum 958 e 969. Biblioteca di Aevum Antiquum: Istituto di Filologia Classica e di Papirologia. Milano: Vita e Pensiero, 2001.

BARCHIESI, Alessandro. Notizie sul «Nuovo Gallo». Atene e Roma, v. n.s. 26, p. 153-66, 1981.

BARCHIESI, Alessandro. Lyric in Rome. In: BUDELMANN, Felix (Ed.). The Cambridge Companion to Greek Lyric. Cambridge: Cambridge University Press, 2009. p. 319-35.

BITTO, Gregor. Lyrik als Philologie. Zur Rezeption hellenistischer Pindarkommentierung in den Oden des Horaz: Rahden/Westf.: VML Verlag Marie Leidorf, 2012.

BULLOCK, Anthony W. Tibullus and the Alexandrians. Proceedings of the Cambridge Philological Society, v. n.s. 19, p. 71-89, 1973.

CAREY, Christopher. Negotiating the public voice. In: DELIGNON, Bénédicte; LE MEUR, Nadine; THÉVENAZ, Olivier (Ed.). La poésie lyrique dans la cité antique. Les Odes d'Horace au miroir de la lyrique grecque archä̈que. Paris: Librairie de Boccard, 2016. p. 177-92. (Collection du CEROR).

CREMONA, Virginio. La poesia civile di Orazio. Milano: Vita e Pensiero, 1982.

D’ALESSIO, Giovan Battista. Introdurione a Callimaco, Inni Epigrammi Ecale. Milano: Rizzoli, 1996. v. I.

DEPEW, Mary. Mimesis and aetiology in Callimachus' Hymns. In: HARDER, M. A; REGTUIT, R. F.; WAKKER, G. C. Callimachus, Hellenistica Groningana I. Groningen: Egbert Forsten, 1993. p. 57-77.

DI LIDDO, Angela Doriana. «Pindarum quisquis studet aemulari» (Hor. Carm. 4, 2). Aufidus, v. 53, p. 21-69, 2004.

DU QUESNAY, I. M. Le M. Horace, Odes 4. 5: Pro reditu Imperatoris Caesaris Divi Filii Augusti. In: HARRISON, Stephen J. (Ed.). Homage to Horace: a bimillenary celebration. Oxford: Oxford University Press, 1995. p. 128-87.

FANTUZZI, Marco; HUNTER, Richard. Muse e modelli. La poesia ellenistica da Alessandro Magno ad Augusto. Roma; Bari: Laterza, 2002. 
FEDELI, Paolo. Properzio. Il Libro terzo delle Elegie. Introduzione, testo e commento. Bari: Adriatica, 1985.

FEDELI, Paolo; CICCARELLI, Irma. Q. Horatii Flacci Carmina Liber IV. Firenze: Le Monnier, 2008.

FRAENKEL, Eduard. Horace. Oxford: Oxford University Press, 1957.

GIANGRANDE, Giuseppe. An alleged Fragment of Gallus. Quaderni Urbinati di Cultura Classica, v. 34, p. 141-53, 1980.

GIANGRANDE, Giuseppe (Ed.). Corolla Londiniensis 2. Amsterdam: J. C. Gieben, 1982.

HARDIE, Philip. Virgil's Aeneid: Cosmos and Imperium. Oxford: Oxford University Press, 1986.

HARRISON, Stephen J. Horace, Pindar, Iullus Antonius, and Augustus: Odes 4. 2. In: HARRISON, Stephen J. (Ed.). Homage to Horace: a bimillenary celebration. Oxford: Oxford University Press, 1995. p. 108-27.

HEKSTER, Olivier. Captured in the Gaze of Power. Visibility, Games and Roman Imperial Representation. In: HEKSTER, Olivier; FOWLER, Richard (Ed.). Imaginary Kings. Royal Images in the Ancient Near East, Greece and Rome. München: Franz Steiner Verlag, 2005.

HEYWORTH, Stephen (Ed.). Sexti Properti Elegi. Oxford: Clarendon Press, 2007.

HEYWORTH, Stephen. Cynthia: A Companion to the Text of Propertius. Oxford: Oxford University Press, 2007.

HOLLIS, Adrian S. Fragments of Roman poetry c.60 BC - AD 20. Oxford: Oxford University Press, 2007.

HUNTER, Richard. Theocritus, Encomium of Ptolemy Philadelphus. Text and translation with introduction and commentary. Berkeley; Los Angeles; London: University of California Press, 2003.

LABATE, Mario. L'arte di farsi amare: modelli culturali e progetto didascalico nell'elegia ovidiana (Biblioteca di 'Materiali e discussioni per l'analisi dei testi classici' II) Pisa: Giardini, 1984.

LYNE, R. O. A. M. Horace. Behind the publicpoetry. New Haven; London: Yale University Press, 1995.

MURGATROYD, Paul. A commentary on the first book of the elegies of Albius Tibullus. Bristol: Bristol Classical Press, 2001 [1980].

NISBET, R. G. M. Some problems of text and interpretation in Horace Odes 3, 14 (Herculis ritu). Papers of the Liverpool Latin Seminar, v. 4, 1983. p. 105-27.

NISBET, R. G. M.; RUDD, Niall. A commentary on Horace. Odes, Book III. Oxford: Oxford University Press, 2004. 
PUTNAM, Michael C. J. Propertius and the New Gallus Fragment. Zeitschrift für Papyrologie und Epigraphik, v. 39, p. 49-56, 1980.

PUTNAM, Michael C. J. Artifices of eternity. Horace's fourth book of Odes. Ithaca-London: Cornell University Press, 1986.

SCHIESARO, Alessandro. Horace's Bacchic Poetics. In: HOUGHTON, L. B. T.; WYKE, Maria. Perceptions of Horace. A Roman Poet and his Readers. Cambridge: Cambridge University Press, 2009. p. 61-79.

STROOTMAN, Rolf. Courts and Elites in the Hellenistic Empires. Edinburgh: Edinburgh University Press, 2014.

SYNDIKUS, Hans Peter. Die Lyrik des Horaz. Eine Interpretation der Oden. Band II, Darmstadt: Wissenschaftliche Buchgesellschaft, 1990.

WACKERNAGEL, Jacob. Über einige antike Anredeformen. Göttingen: Kaestner, 1912 (= Kleine Schriften, Göttingen: Vandenhoeck \& Ruprecht, 1955, II).

WATSON, Lindsay C. A Commentary on Horace's Epodes. Oxford: Oxford University Press, 2003. 
